\title{
Exploring the social position of tactile maps in Japan
}

\author{
Masahiro Tanaka ${ }^{\mathrm{a}}$ \\ a JSPS Research Fellow, Nagoya University, rop2054@gmail.com
}

Keywords: Tactile map, Visually impaired people, Materiality

\begin{abstract}
:
It seems that the aim of conventional studies on tactile maps for visually impaired people have been to improve their utility in terms of instrumentalism. However, given the recent progress of disability studies in social science and postrepresentational approach in recent map studies, it is necessary to examine how tactile maps work in the society and how they relate to "disability" as a social phenomenon. These are also important issues to think about how geospatial information technology for visually impaired people embed in the society. Hence, regarding the map as more-thanrepresentation, this paper analysed various documents (e.g. newspaper articles, essays, instruction manuals for orientation and mobility specialist), and considered the social position of tactile maps in Japan. The results showed the following.
\end{abstract}

The tactile map has a long history as a teaching material of geography. It was used for education for the blind in Western countries in the late 18th century. This situation was introduced to Japan by overseas memoir. For example, in the late Edo era, Namura Gohachirou who was a member of Japanese Embassy to the United States witnessed geography education using tactile maps at the blind school in New York. In his diary named Akou Nikki, Namura said that the education for blinds using tactile maps seemed to "translation" from the words of those who can see both eyes well to the words of visually impaired people. His statement clearly shows the material difference of tactile maps. Many tactile maps have acquired social status as "translated objects". The material form of tactile maps is different from the "map" (visual map) known to the general public, so it makes awareness of physical differences between the body of visually impaired people and sighted people. In 1880, the first blind school in Japan, Kyoto Moua In had exhibited teaching materials and other instruments to the exposition, including a tactile map representing the city of Kyoto. Such a social event also had a role to attract the attention of sighted people to the material heterogeneity of the tactile map and visually impaired people.

The 1960s-80s was the period of the situation of tactile maps changed significantly in Japan. In the 1960s, rehabilitation techniques for visual impairments was introduced to Japan from USA, and the concept of "orientation" which was lacking in conventional walking training diffused. In accordance with these movement, the teaching manual of orientation and mobility $(\mathrm{O} \& \mathrm{M})$ training became write the methodologies to make and use the tactile map for the training. In 1964, Kazuo Honma, the founder of Japan Brail Library, visited all over the world and bought lots of tools for visually impaired people, including tactile maps, at the blind libraries in various places and brought them back to Japan. He held an exhibition to show those materials in the following year. Furthermore, as the International Year of Disabled Persons 1981 and the subsequent enforcement of various laws related to people with disability, it has been emphasized to create cities where physically impaired people can go out of their homes. Since that time the tactile maps began to be installed in public facilities, and were introduced in the assistive technology catalogs. In this way, the tactile map was incorporated into the context of "outdoor behavior" and "walking". Not only the body of visually impaired people but also the tactile maps as material objects have increasingly been exposed to the "outside" spaces (e.g. city, road). As a result, the tactile map became understood from the viewpoint of "safety", and it became involved with human and non-human actors (e.g. government offices, volunteer organizations, barrier-free laws, traffic guidelines) different from those of geography education. 